This item was submitted to Loughborough's Research Repository by the author.

Items in Figshare are protected by copyright, with all rights reserved, unless otherwise indicated.

\title{
Tunable oxygen defect density and location for enhancement of energy storage
}

PLEASE CITE THE PUBLISHED VERSION

https://doi.org/10.1016/j.jechem.2020.12.016

PUBLISHER

Elsevier BV

VERSION

AM (Accepted Manuscript)

\section{PUBLISHER STATEMENT}

This paper was accepted for publication in the journal Journal of Energy Chemistry and the definitive published version is available at https://doi.org/10.1016/j.jechem.2020.12.016.

LICENCE

CC BY-NC-ND 4.0

\section{REPOSITORY RECORD}

Chen, Jun, Jiangao Li, Ling Sun, Zhong Lin, Zhengguang Hu, Hongtao Zhang, Xiaoling Wu, Dongbo Zhang, Guoan Cheng, and Ruiting Zheng. 2020. "Tunable Oxygen Defect Density and Location for Enhancement of Energy Storage”. Loughborough University. https://hdl.handle.net/2134/13514494.v1. 


\section{Tunable oxygen defect density and location for enhancement of energy storage}

Jun Chen ${ }^{\mathrm{a}}$, Jiangao Li ${ }^{\mathrm{a}}$, Ling Sun ${ }^{\mathrm{a}}$, Zhong Lin ${ }^{\mathrm{a}}$, Zhengguang $\mathrm{Hu}^{\mathrm{b}}$, Hongtao Zhang ${ }^{\mathrm{c}}$, Xiaoling $\mathrm{Wu}^{\mathrm{a}}$, Dongbo Zhang ${ }^{\mathrm{a}, \mathrm{d}}$, Guoan Cheng ${ }^{\mathrm{a}}$, Ruiting Zheng, ${ }^{\mathrm{a}}$ *

${ }^{a}$ Key Laboratory of Radiation Beam Technology and Materials Modification of the Ministry of Education, College of Nuclear Science and Technology, Beijing Normal University, Beijing 100875, China

${ }^{\mathrm{b}}$ Department of Physics, Nanchang University, Nanchang 330031, Jiangxi, China

${ }^{\mathrm{c}}$ Department of Materials, Loughborough University, Leicestershire, LE11 3TU, UK

d Beijing Computational Science Research Center, Beijing 100193, China

${ }^{*}$ Corresponding author.

E-mail address: rtzheng@bnu.edu.cn (R. Zheng)

\section{ABSTRACT}

Defect engineering is in the limelight for the fabrication of electrochemical energy storage devices. However, determining the influence of the defect density and location on the electrochemical behavior remains challenging. Herein, self-organized $\mathrm{TiO}_{2}$ nanotube arrays (TNTAs) are synthesized by anodization, and their oxygen defect location and density are tuned by a controllable post-annealing process. TNTAs annealed at $600{ }^{\circ} \mathrm{C}$ in $\mathrm{N}_{2}$ exhibit the highest capacity (289.2 $\mathrm{mAh} \mathrm{g}^{-1}$ at $0.8 \mathrm{C}$ ) for lithium-ion storage, while those annealed at $900{ }^{\circ} \mathrm{C}$ in $\mathrm{N}_{2}$ show a specific capacitance of $35.6 \mathrm{mF} \mathrm{cm}^{-2}$ and stability above $96 \%$ after 10,000 cycles for supercapacitor. Ex situ electron paramagnetic resonance spectra show that the surface-exposed oxygen defects increase, but the bulk embedded oxygen defects decrease with increasing annealing temperature. Density functional theory simulations reveal that a higher density of bulk oxygen defects corresponds to higher localized electrons states, which upshift the Fermi level and facilitate the lithium intercalation kinetic process. Meanwhile, differential charge density calculation indicates that the increase of surface oxygen defects in the anatase (101) plane leads to higher density excess electrons, which act as negative charge centers to enhance the surface potential for ion adsorption. This oxygen-deficient location and density tunable 
strategy introduce new opportunities for high-energy and high-power-density energy storage systems.

Keywords: Oxygen defects; Excess electrons; Supercapacitors; Lithium-ion batteries; DFT calculations

\section{Introduction}

Lithium-ion battery (LIB) and supercapacitor (SC) technologies are two dominant and complementary means of electrochemical charge storage to satisfy the increasing demand for portable electronics, electric vehicles, and smart grids [1-4]. The key requirements to be met in the development of electrode materials for these two kinds of charge storage devices are high energy density, high power density, and high stability [5-8]. Defect engineering is a powerful strategy to enhance the electrochemical energy storage properties.

Titanium dioxide $\left(\mathrm{TiO}_{2}\right)$ is a competitive electrode material used in LIBs and SCs devices owing to its high structural stability, natural abundance of source elements, low cost, and high theoretical capacity [9-12]. Among the available $\mathrm{TiO}_{2}$ nanomaterials, self-organized $\mathrm{TiO}_{2}$ nanotube arrays (TNTAs) synthesized by anodization can be excellent candidates as an active electrode in LIBs and SCs $[13,14]$. The unique one-dimensional (1D) nanotube structure can provide a large surface area and thus, more active sites for ion storage, and can accelerate electron and ion transport [15]. Meanwhile, the binder- and additive-free properties can prevent interface issues during charge storage, making these arrays an excellent candidate for the analysis of the charge storage process $[16,17]$. However, due to their intrinsically sluggish electronic and ionic diffusion kinetics, $\mathrm{TiO}_{2}$-based electrode materials need further modification before use in high-performance energy storage devices [18-20]. The introduction of oxygen defects is the most widely used method among those currently available for this purpose. Oxygen defects can not only tune the optical and photocatalytic properties of $\mathrm{TiO}_{2}$ [21], but also improve its electrochemical charge storage behavior by enhancing the electronic and ionic 
conductivity of $\mathrm{TiO}_{2}$. However, in comparison with $\mathrm{TiO}_{2}$ nanoparticles, only few studies have focused on defects related to charge storage devices based on 1D TNTAs. Wu et al. investigated the enhancement in the SC performance with TNTAs resulting from the introduction of oxygen vacancies by $\mathrm{NaBH}_{4}$ reduction [22]. Naeimeh et al. demonstrated that the electrochemical reduction of TNTAs can improve the SC performance due to the formation of oxygen vacancies [23]. Xiong et al. found that defect generation by annealing in a different atmosphere can enhance the lithium-ion storage properties of TNTAs [24].

In previous research [25-27], it was found that the bulk and surface defect ratio have a significant impact on the photocatalytic performance of $\mathrm{TiO}_{2}$ nanomaterials. However, controllable tuning of the oxygen defect location and density in TNTAs remains challenging. The relationship of the defect location and density with electrochemical charge storage remains ambiguous. Therefore, it is important to optimize the density and location of oxygen defects and clarify the contribution of different defects in charge storage for future electrode design. In the present work, the oxygen defect density and location are systematically tuned by a controllable post-annealing process. We found that the surface-exposed oxygen defect density increases with temperature, whereas the bulk embedded oxygen defect density decreases accordingly. The introduction of oxygen defects in the bulk and surface will enhance the localized electron density around the adjacent $\mathrm{Ti}$ atom $\left(\mathrm{Ti}^{3+}\right.$ form $)$ in both, the bulk and surface. The excess electrons in the bulk interact with the inserted lithium ions and improve the intercalation and diffusion process. Meanwhile, the excess electrons upshift the Fermi level to accelerate the electron transportation. Therefore, the introduction of high density bulk oxygen defects is effective in fabricating high-performance LIBs. TNTAs annealed at $600{ }^{\circ} \mathrm{C}$ in $\mathrm{N}_{2}$ exhibit the highest bulk oxygen defect density, highest capacity of $289.2 \mathrm{mAh} \mathrm{g}^{-1}$ at $0.8 \mathrm{C}$, and provide the best performance in this work. Similarly, the excess electrons on the surface also introduce excess electrons in the anatase (101) plane and enhance the surface potential, as 
verified by Kelvin probe force microscopy (KPFM) characterization. Owing to the interaction between the excess electrons and surface sodium ions, the higher surface excess electrons result in a faster sodium ion adsorption kinetic process in the anatase (101) plane. Therefore, a higher surface oxygen defect density will improve the performance of the surface-charge-processdominated energy storage device. The TNTAs annealed at $900{ }^{\circ} \mathrm{C}$ in $\mathrm{N}_{2}$ possess the highest surface oxygen defect density, and yield an excellent specific capacitance of $35.6 \mathrm{mF} \mathrm{cm}{ }^{-2}(100$ $\mathrm{mV} \mathrm{s}^{-1}$ ), which is the highest value ever reported. Moreover, cyclic voltammetry (CV) kinetic analysis confirms that both, pseudocapacitive and diffusion-controlled storage mechanisms, occur in the LIB. Further, the pseudocapacitive effect is enhanced with increasing density of the surface-exposed oxygen defects, in accordance with the surface charge storage mechanism in an SC device. The defect design strategy in this work is briefly summarized in Scheme 1 .

\section{Experimental}

\subsection{Materials}

All the chemicals (AR) were purchased from Xilong Chemical Co., Ltd. without further purification. The titanium foil was washed with acetone, ethanol, and deionized water for 10 mins before anodization.

\subsection{Sample preparation and electrocheical tests}

In a typical procedure, the titanium foil (anode) and platinum foil (cathode) were used as two electrodes in the $\mathrm{F}^{-}$contained electrolyte $\left(1 \mathrm{~g} \mathrm{NH}_{4} \mathrm{~F}, 6 \mathrm{~mL} \mathrm{H}_{2} \mathrm{O}, 6 \mathrm{~mL} \mathrm{H}_{3} \mathrm{PO}_{4}\right.$, and $187 \mathrm{~mL}$ glycol) with an anodization voltage of $80 \mathrm{~V}$. After 120 min of anodization, the as-anodized $\mathrm{TiO}_{2}$ film was removed using an ultrasonic cleaner followed by the second anodization with the same synthesis parameters. The as-obtained TNTA films were washed with deionized water before annealing in $\mathrm{N}_{2}$. The $\mathrm{N}_{2}$ annealing process was conducted in a tube furnace with an $\mathrm{N}_{2}$ flux of $200 \mathrm{sccm}$ for $1 \mathrm{~h}$ at different annealing temperatures $\left(600-900{ }^{\circ} \mathrm{C}\right)$. For the LIB test, lithium foil was used as the anode and the TNTAs (cut into $1 \mathrm{~cm}^{2}$ ) were used as the cathode with 
Celgard 2400 film as the separator in a CR 2025 coin cell. The electrolyte (Kunlunchem, China) consisted of $1 \mathrm{M} \mathrm{LiPF}_{6}$ dissolved in a 1:1 (volume ratio) mixture of ethylene carbonate (EC) and diethyl carbonate (DEC) with an addition of fluoroethylene carbonate (FEC). The battery assembly process was conducted in a glove box with a $\mathrm{H}_{2} \mathrm{O} / \mathrm{O}_{2}$ content lower than $1 \mathrm{ppm}$. For the supercapacitor performance test, a three-electrode test method was used with $0.5 \mathrm{M} \mathrm{Na}_{2} \mathrm{SO}_{4}$ aqueous solution as the electrolyte. The TNTAs were used as the working electrode, the platinum wire served as the counter electrode, and $\mathrm{Ag} / \mathrm{AgCl}(3 \mathrm{M} \mathrm{KCl})$ served as the reference electrode. The material characterization details, electric/electrochemical test details, and DFT calculation methods are elucidated in the Supporting Information.

\section{Results and discussion}

To better highlight the influence of intrinsic bulk or surface oxygen defects on the charge storage properties of electrode materials, self-supported TNTAs are used as the charge storage electrode, to eliminate the requirement of (and hence, obstruction by) a binder and additive. Fig. 1(a) illustrates a facile synthesis procedure for the black TNTAs. A typical two-step electrochemical anodization method is employed to obtain the as-anodized TNTAs [28]. Subsequently, facile and controllable post-annealing is conducted in $\mathrm{N}_{2}$ to tune the oxygen defect location and density of the black TNTAs. Scanning electron microscopy (SEM) images of the as-anodized TNTAs in Fig. 1(b-f) reveal that the average inner diameter of the tube is approximately $100 \mathrm{~nm}$ and the length of the tube is approximately $10 \mu \mathrm{m}$. Fig. 1(g and h) shows the typical TEM images of surface and cross-section in TNTAs which can also confirm the diameter is approximately $100 \mathrm{~nm}$. The high-resolution transmission electron microscopy (HRTEM) image in Fig. 1(i) and the selected area electron diffraction (SAED) image in Fig. 1(j) both illustrate the amorphous phase of the as-anodized TNTAs. X-ray diffraction (XRD) was conducted to further demonstrate the crystal structure and phase compositions of the 
TNTAs annealed in $\mathrm{N}_{2}$ at $600-900{ }^{\circ} \mathrm{C}$, as shown in Fig. 2(a). All TNTAs annealed in $\mathrm{N}_{2}$ mainly show an anatase phase with A (101), A (200), and A (004) as the major diffraction peaks. It should be noted here that only few rutile-phase diffraction peaks can be observed in the TNTAs annealed in $\mathrm{N}_{2}$ at $800-900{ }^{\circ} \mathrm{C}$. Moreover, the enlarged A (101) peak shifts to larger angles with increasing annealing temperature, signifying a decrease in the bulk oxygen defects [29]. This phenomenon can be attributed to the introduction of oxygen vacancies which lead to the formation of low valence state species ( vacancies. In the $\mathrm{TiO}_{2}$ lattice, the size variation in $\mathrm{Ti}^{4+}$ with $\mathrm{Ti}^{3+}$ and the oxygen vacancies distort the local symmetry of the $\mathrm{TiO}_{2}$ lattice and generate strain in the lattice ${ }^{[30,31]}$. By undergoing lattice expansion, the strain can be released in the $\mathrm{TiO}_{2}$ lattice ${ }^{[32]}$. Therefore, with the increase in bulk oxygen defects, the XRD peak position shifts to the smaller diffraction angle direction (lattice plane expansion). Raman spectra were acquired to further reveal the oxygen defects in the TNTAs, as shown in Fig. 2(b). All the TNTAs annealed in $\mathrm{N}_{2}$ show typical bands (3Eg, A1g, and 2B1g) of anatase, and the Raman scattering intensity decreases with decreasing annealing temperature. This phenomenon can also confirm an increase in the oxygen defect density, which is in agreement with XRD results. The SEM and TEM images of the TNTAs annealed in $\mathrm{N}_{2}$ are shown in Fig. 2(c-f) and $(\mathrm{g}-\mathrm{j})$, respectively, and illustrate that no distinct morphology change occurs during post-annealing. Fig. 2(k-n) shows the HRTEM images and the insets are the corresponding SAED images, which verify that the anatase phase of all post-annealed TNTAs is in accordance with the XRD and Raman spectra. It should be noted here that the A (101) plane lattice distance variation trend measured by HRTEM may not match well with the XRD results. This is because HRTEM characterization is a high magnification localized method that can only provide the localized lattice information of $\mathrm{TiO}_{2}$. In contrast, $\mathrm{XRD}$ is a macroscopic statistical characterization technique that can provide the entire bulk information of the sample. Consequently, this may be the main reason why the XRD 
results and the HRTEM results do not match well, particularly when a few HRTEM images are used. From the structural analysis above, we can conclude that except for the defect states, the small structure and difference in morphology may not be the contributing factors to the properties of the TNTAs.

Because the XRD and Raman spectra can only provide qualitative information of oxygen defects, more accurate information on the oxygen defect location and density signal are obtained from electron paramagnetic resonance (EPR) spectra based on unpaired electrons, as shown in Fig. 3(a-d). Fig. S1(a and b) shows the enlarge EPR spectra corresponding to the black rectangle region in Fig. 3( $\mathrm{a}$ and $\mathrm{b}$ ). All the TNTAs annealed in $\mathrm{N}_{2}$ show two typical resonance peaks with $g$ factors of 1.99 and 1.93, respectively. These two resonance peaks can be attributed to the lattice-embedded oxygen defects $(g=1.99)$ and surface-exposed oxygen defects $(g=1.93)$. By calculating the spin density of the corresponding resonance peaks using two times integral method [25], we can semi-quantitatively determine the oxygen defect density, as shown in Fig. 3(e and f). The surface-exposed oxygen defect density increases with increasing annealing temperature, whereas the lattice-embedded oxygen defect density decreases with increasing annealing temperature. In particular, $\mathrm{N}_{2}-800{ }^{\circ} \mathrm{C}$ sample shows a sharply decreasing latticeembedded oxygen defect density, which may be due to the partial phase transition from anatase to rutile, as shown in the XRD spectra. Moreover, the $\mathrm{N}_{2}-900{ }^{\circ} \mathrm{C}$ sample only shows the surfaceexposed oxygen defect signal, and no lattice-embedded oxygen defect signal can be detected in the EPR spectra. Because the initial TNTAs are amorphous, the generation of a different defect state under a different annealing temperature may be caused by simultaneous phase transition and oxygen defect generation, which has been verified in our previous work [33]. A higher annealing temperature will accelerate crystallization in the embedded lattice, and oxygen atoms will be fixed in the embedded lattice more quickly to avoid oxygen defect generation in bulk in the initial annealing stage [34]. Therefore, a higher temperature will lead to a lower density of 
bulk oxygen defects. However, because the surface oxygen atoms in the surface lattice need less energy to escape the surface, a higher temperature will lead to a higher density of surface oxygen defects. From the distinct differences in defect states of these four samples, we can confirm the exact beneficial role of different oxygen defect locations and densities by studying their corresponding electrochemical properties.

To verify the effects of the bulk and surface defects on the dominant bulk charge storage process, we prepared LIBs using different TNTA materials as anode electrodes. Lithium half cells were assembled and their electrochemical capacities were evaluated by galvanostatic charge and discharge (GCD) cycle tests with a potential window of 0.01-3 V. Fig. 4(a) shows that the $\mathrm{N}_{2}-600,700,800$, and $900{ }^{\circ} \mathrm{C}$ TNTAs exhibit high cycle stability with reversible capacities of $289.17,236.59,162.99$, and $141.96 \mathrm{mAh} \mathrm{g}^{-1}$, respectively, after 100 cycles at a high current density of $262.88 \mathrm{~mA} \mathrm{~g}^{-1}(\sim 0.8 \mathrm{C})$. The enhancement in the lithium-ion storage capacity should be due to the remarkable increase in the bulk oxygen defect density with decreasing annealing temperature. An increase in the density of bulk oxygen defects will introduce excess electrons around the adjacent $\mathrm{Ti}$ atom to ease the insertion of more lithium ions into the $\mathrm{TiO}_{2}$ lattice [35]. Fig. 4(b) illustrates how oxygen defects influence lithium diffusion in the bulk according to the rate performance of all TNTAs, with current densities varying from $0.8 \mathrm{C}$ to $8 \mathrm{C}$. It is observed that the rate performance is enhanced by increasing the bulk oxygen defect density. The lithium-ion storage performances of different $\mathrm{TiO}_{2}$ nanotube array-based LIBs in recent years are presented in Table S1. The TNTAs in this work exhibit outstanding electrochemical performance due to the remarkable enhancement of the ultra-high density bulk oxygen defect. Electrochemical impedance spectroscopy (EIS) plots were also obtained after 20 cycles to further reveal the electrochemical process in the interface 
and the bulk, as shown in Fig. 4(c). The EIS plots show a semicircle and a straight line, which correspond to the charge transfer $\left(R_{\mathrm{ct}}\right)$ and solid-state bulk diffusion of lithium ions $\left(R_{\mathrm{d}}\right)$, respectively. After fitting the EIS plots using the equivalent circuit depicted in the inset of Fig. 4(c) (the fitting parameters are summarized in the Table S2), $R_{\mathrm{ct}}$ and $R_{\mathrm{d}}$ show a decrease with increasing bulk oxygen defects, implying a gradual increase in the charge transfer conductivity and ion diffusion kinetic process. Moreover, we calculated the diffusion coefficient of the lithium ions according to the slope of the linear fitting of $Z_{\text {re }}$ vs. $\omega^{-1 / 2}$ using the following equation $[36,37]$ :

$$
D_{L i^{+}}=\frac{1}{2}\left(\frac{R T}{S n^{2} F^{2} C A}\right)^{2},
$$

where $R$ is the gas constant, $T$ is the absolute temperature, $S$ is the surface area, $n$ is the number of transferred electrons per molecule during the electrochemical reaction, $F$ is Faraday's constant, $C$ is the lithium-ion concentration, and $A$ is the Warburg factor, which is the slope of the linear fitting of $Z_{\text {re }}$ vs. $\omega^{-1 / 2}$. The fitting results and lithium-ion diffusion coefficient are depicted in Fig. S2. The lithium-ion coefficient increases with the increase in bulk oxygen defect density, indicating that the oxygen defect can accelerate the lithium-ion diffusion rate in the TNTA bulk electrodes. Galvanostatic intermittent titration technique (GITT) tests were also performed to determine the lithium-ion diffusion coefficient in the TNTA electrodes, as shown in Figs. 4(d) and S3(a-d). The $\mathrm{Li}^{+}$diffusion coefficient can be calculated according to Eq. (2) [38-41]:

$$
D\left(\mathrm{Li}^{+}\right)=\frac{4}{\pi \tau}\left(\frac{m V_{\mathrm{m}}}{M A}\right)^{2}\left(\frac{\Delta E_{\mathrm{s}}}{\Delta E_{\tau}}\right)^{2}
$$

where $D\left(\mathrm{Li}^{+}\right)$is the diffusion coefficient of the lithium ions, $\tau$ is the pulse time, $m$ is the mass of active material, $V_{\mathrm{m}}$ is the molar volume, $M$ is the molar mass, $A$ is the area of the electrode, $\Delta E_{\mathrm{s}}$ and $\Delta E_{\tau}$ can be calculated from the $V-t$ plots in Fig. 4(d). 
The diffusion coefficient as a function of the applied voltage is shown in Fig. 4(e). The diffusion coefficient increases with increasing bulk oxygen defect density, which is in agreement with the EIS results. Thus, we can conclude that bulk embedded oxygen defects will lead to a higher lithium diffusion coefficient in the bulk of the $\mathrm{TiO}_{2}$ lattice and thus improve the rate performance. The galvanostatic charge/discharge curves of the $\mathrm{N}_{2}-600{ }^{\circ} \mathrm{C}$ TNTAs at different rates are depicted in Fig. 4(f), and those of the other samples are shown in Fig. S4(a-d), from which we see that the insertion/desertion plateaus of all TNTAs are located around 1.6 and 2.1 V. These plateaus are consistent with the initial five cycles in the CV results depicted in Fig. 4(g). The initial cycle in the CV curve shows three main peaks corresponding to the lithiation potential, at $1.5,1.2$, and $0.9 \mathrm{~V}$, which may be attributed to the decomposition of the electrolyte and the formation of the SEI (to stabilize the interface); the potential remains at $1.6 \mathrm{~V}$ in the subsequent cycles. The sustained cycle stability indicated by the CV curves and GCD plots can confirm the stable structure of TNTAs during lithium insertion and extraction, which implies that TNTAs can serve as an excellent anode material for bulk charge storage devices.

To further investigate the charge storage mechanism of all the TNTAs and reveal the surface pseudocapacitive contribution (which may be related to the surface oxygen defects), CV kinetic analysis was conducted. Fig. $4(\mathrm{~h})$ shows the $\mathrm{CV}$ curves of the $\mathrm{N}_{2}-600{ }^{\circ} \mathrm{C}$ TNTAs at $0.1,0.2$, $0.5,1,2$, and $5 \mathrm{mV} \mathrm{s}^{-1}$. It is well known that the $\mathrm{CV}$ curve represents the total charge storage capacity of a $\mathrm{TiO}_{2}$ anode-based LIB, which can be divided into contributions from the diffusioncontrolled capacity and surface-dominated pseudo-capacity by Dunn's method [33]. The measured current and scan rate satisfy Eq. (3) [42]:

$$
i=a v^{b},
$$

where $i$ is the current, $a$ is a constant, $v$ is the scanning voltage, and the $b$ value can be fitted by the slope of the $\log (i)-\log (v)$ plots. If the $b$ value is equal to 0.5 , it implies that the charge storage mechanism is diffusion-controlled. A $b$ value of 1 indicates the pseudocapacitive 
storage mechanism. Fig. 4(i) shows the anodic and cathodic $\log (\mathrm{I}$ peak $)-\log (v)$ plots of the $\mathrm{N}_{2}-$ $600{ }^{\circ} \mathrm{C}$ TNTAs. The $b$ values of the anodic and cathodic peaks are 0.542 and 0.618 (located between 0.5 and 1), respectively. Therefore, we can confirm that the charge storage mechanism of $\mathrm{N}_{2}-600{ }^{\circ} \mathrm{C}$ TNTAs can be attributed to both, surface pseudocapacitive charge storage and diffusion-controlled bulk charge storage. To quantitatively calculate the contribution differences of these two processes, Eq. (4) is applied [43]:

$$
i=k_{1} v+k_{2} v^{0.5}
$$

In Eq. (4), $k_{1}$ and $k_{2}$ represent coefficients, and $i(V), k_{1} v$, and $k_{2} v^{0.5}$ represent the total charge storage, surface-dominated pseudocapacitive charge storage, and diffusion-controlled charge storage, respectively. The relationship between $i(V)$ and scan rate $v$ at different potentials during $\mathrm{CV}$ from 0.1 to $5 \mathrm{mV} \mathrm{s}^{-1}$ is used to fit $k_{1}$ and $k_{2}$. According to the values of $k_{1}$ at different potentials, the potential profile for the current from the pseudocapacitive-dominated contribution (pink region) at $0.2 \mathrm{mV} \mathrm{s}^{-1}$ is approximately $24.64 \%$ as depicted in Fig. 4(j). Similarly, the pseudocapacitive contributions of other TNTAs are shown in the Fig. S5(a-i), and the pseudocapacitive contribution ratio at $0.2 \mathrm{mV} \mathrm{s}^{-1}$ is provided in Fig. $4(\mathrm{k})$. Furthermore, the $\mathrm{N}_{2}-900{ }^{\circ} \mathrm{C}$ sample, which only possesses surface-exposed oxygen defects without latticeembedded oxygen defects, shows the highest pseudocapacitive contribution ratio. This indicates that the pseudocapacitive contribution will gradually increase with increasing density of the surface-exposed oxygen defects. The enhanced surface pseudocapacitive reaction reveals that the surface-exposed oxygen defects may be active faradaic sites for surface-dominated charge storage. The introduction of oxygen defects increases the excess electrons around the adjacent titanium atoms $[44,45]$. These excess electrons can not only increase the electronic and ionic conductivity but also act as negative active centers to boost the lithium-ion storage sites. Moreover, the removal of oxygen atoms from the lattice can provide more space for the storage and diffusion of lithium ions. Furthermore, the oxygen defect in the surface can boost the 
surface pseudocapacitive reactive sites and enhance the surface lithium storage capacity to a certain extent

Following the increase in the surface pseudocapacitive reaction sites from the surface-exposed oxygen defects in the LIB, we further find that the $\mathrm{N}_{2}$-annealed black TNTAs can also be used as an excellent ion storage electrode in an SC electrode. The electrochemical properties of TNTAs with different oxygen defect locations and densities were evaluated by CV and GCD in a typical three-electrode system in $0.5 \mathrm{M} \mathrm{Na}_{2} \mathrm{SO}_{4}$ electrolyte.

Figs. 5(a) and S6(a-d) show the CV curves of all the annealed TNTAs, acquired at various scanning rates ranging from 10 to $1000 \mathrm{mV} \mathrm{s}^{-1}$. The $\mathrm{CV}$ curves of all the $\mathrm{N}_{2}$-annealed TNTAs remain unchanged (rectangular shape) without redox peaks when the scanning rate is increased from 10 to $1000 \mathrm{mV} \mathrm{s}^{-1}$ with a potential window from -0.6 to $0.5 \mathrm{~V}$ (vs. $\mathrm{Ag} / \mathrm{AgCl}$ ). Fig. 5(b) displays the GCD curves of all SC electrodes, which show a triangular shape over a wide range of current densities from 0.2 to $1 \mathrm{~mA} \mathrm{~cm}^{-2}$. The $\mathrm{CV}$ curves and GCD plots both indicate excellent electrochemical double layer capacitor behavior based on the ion adsorption/desorption on the TNTAs. The rate performance obtained from the CV curves of all the TNTAs is summarized in Fig. 5(c). The capacity increases due to an increase in the surfaceexposed oxygen defect density. This implies that surface-exposed oxygen defects may be the active surface ion adsorption sites in surface charge storage. The increase in surface oxygen defects introduces excess electrons around the adjacent $\mathrm{Ti}$ atom to ease the adsorption of sodium ions on the $\mathrm{TiO}_{2}$ lattice surface due to the interaction of electrons and ions [46]. To evaluate the charge storage reversibility of all the $\mathrm{N}_{2}$-annealed $\mathrm{SC}$ electrodes, $\mathrm{CV}$ retention curves are shown in Figs. 5(d) and S6(e-h). All the SC electrodes show extremely high stability without an apparent reduction in capacity after $10,000 \mathrm{CV}$ cycles at $100 \mathrm{mV} \mathrm{s}^{-1}$. Details on the capacity retention are summarized in Fig. 5(e). The initial capacities of the $\mathrm{N}_{2}-600,700,800$, and $900{ }^{\circ} \mathrm{C}$ annealed samples can reach as high as $11.4,18.5,23.9$, and $35.6 \mathrm{mF} \mathrm{cm}^{-2}$, respectively. 
Furthermore, the capacity retentions of the $\mathrm{N}_{2}$-annealed TNTAs can reach as high as $91.94 \%$, $95.74 \%, 94.09 \%$, and $96.04 \%$, respectively, indicating the ultra-stable nature of the SC electrodes.

To further understand the improved surface-related electrochemical behavior of the surfaceexposed oxygen-defect-abundant TNTAs electrodes, EIS measurements were carried out in a frequency range from $100 \mathrm{kHz}$ to $0.1 \mathrm{~Hz}$. The Nyquist plots of all three electrodes display semicircles in the high-frequency region followed by a straight tail in the low-frequency region, which is associated with charge transfer and ionic diffusion, respectively. The $Z_{\mathrm{re}}-\omega^{-1 / 2}$ plots of EIS in the supercapacitor can be linearly fitted in the diffusion region, as depicted in Fig. S7. The slope of the $Z_{\mathrm{re}}-\omega^{-1 / 2}$ plots decreases with increasing surface-exposed oxygen defect density, implying improved ionic diffusion on the surface of the TNTA electrodes. Furthermore, the equivalent circuit (upper inset of Fig. 5(f)) was adopted to fit the Nyquist plots and the fitting parameters are summarized in Table S3, where the intercept on the real axis represents the internal resistance $\left(R_{\mathrm{S}}\right)$ of the entire system including electrode materials, electrolyte, current collector, and the corresponding contact resistance. $R_{\mathrm{S}}$ and the charge transfer resistance $\left(R_{\mathrm{ct}}\right)$ will decrease with increasing surface-exposed oxygen defect density, indicating the improved electrical conductivity and enhanced charge storage kinetics. The solid-state electronic conductivity was also measured and is shown in Fig. 5(g) and Table S4. The enhanced electronic conductivity is in agreement with the EIS fitting results, which can further confirm the improved SC performance with increasing surface oxygen defect density. From the discussion above, we can conclude that surface oxygen defects will be responsible for the improvement in surface electronic and ionic transfer, and consequently, improved surfacerelated ion storage. Moreover, as depicted in Fig. 5(h), the $\mathrm{N}_{2}-900{ }^{\circ} \mathrm{C}$ TNTA-based SCs achieve the best area capacity rate performance in comparison with the $\mathrm{TiO}_{2}$ nanotube array-based SC electrode in the literature $[13,14,22,47-51]$. 
To obtain further insights on the surface charge storage enhancement induced by surface oxygen defects, the surface potential of the TNTAs was determined by KPFM. Surface oxygen defects will significantly influence the electronic structure and surface energy level of $\mathrm{TiO}_{2}$. The surface electric property will also considerably affect the surface ion adsorption/desorption active sites and the kinetic process. Fig. $6\left(\mathrm{a}, \mathrm{c}\right.$, e and g) shows the surface morphology of the $\mathrm{N}_{2}$-annealed TNTAs, and Fig. 6(b, d, f and h) shows the corresponding contact potential difference (CPD) mapping images. The summarized result of the potential mapping is shown in Fig. 6(i). The CPD was calculated from following equation [52]:

$$
\mathrm{CPD}=\Phi_{\text {tip }}-\Phi_{\text {sample }}
$$

Consequently, we can conclude that the surface potential will increase with increasing surfaceexposed oxygen defect density. A higher surface potential indicates a higher Fermi level in the bandgap, which will reduce the electron movement barrier on the surface and enhance the charge transfer and storage capabilities. The enhancement in surface potential and surface electronic conductivity will improve the surface charge storage kinetic process.

The specific surface area and crystalline phase may also affect the electrochemical properties of electrode materials [53]. However, herein, the morphology and crystalline phase change of the $\mathrm{TiO}_{2}$ nanotube arrays under different annealing conditions is not evident according to the structural and morphological characterizations. Moreover, the oxygen defect location and density of TNTAs change significantly, which should be considered as the main factors in determining the electrochemical properties of TNTAs.

To further understand the mechanisms of the bulk and surface charge storage enhancement derived from the bulk and surface oxygen defects, DFT calculations were conducted to understand the $\mathrm{Li}^{+}$insertion/extraction and $\mathrm{Na}^{+}$adsorption/desorption processes in $\mathrm{TiO}_{2}$ with different densities of oxygen defects. For lithium-ion storage, the original $2 \times 2 \times 1$ anatase supercells contained $16 \mathrm{Ti}$ and $32 \mathrm{O}$ atoms. After removing $n(n=0,1(\sim 2.1 \%), 2(\sim 4.2 \%)$, 
and $3(\sim 6.3 \%))$ oxygen atoms from the anatase lattice, the $\mathrm{Ti}$ and $\mathrm{O}$ atoms contributed DOSs and the total DOSs are depicted in Fig. $7(\mathrm{a}-\mathrm{d})$. The insets are the corresponding crystals structures. All four types of supercell show similar band structures; the $\mathrm{O}$ atoms mainly contribute to the $\mathrm{CB}$, while the Ti atoms primarily contribute to the valence band (VB). After introducing oxygen vacancies into the anatase lattice, the Fermi level shifts to the $\mathrm{CB}$, which implies the increase in electronic conductivity. Furthermore, after introducing oxygen vacancies, the $\mathrm{CB}$ shows an enhanced small peak near the VB in the bandgap, which is a typical donor level. With increasing oxygen defect density, the intensity of the small peak below the CB increases gradually, which indicates an increase in the donor energy level derived from the increase in excess electrons around the oxygen defects. Fig. 7(e-h) depicts the DOSs of lithiation anatase with different densities of oxygen defects. For the anatase with oxygen vacancies, the small peaks below the $\mathrm{CB}$ increase significantly while no change is observed for the anatase without oxygen vacancies. This phenomenon indicates that the oxygen vacancies can interact with the inserted lithium ion. To further understand this interaction process, the differential charge densities of anatase $\mathrm{TiO}_{2}$ with different concentrations of oxygen vacancies are calculated as depicted in Fig. 8(b-d). With the increase in the oxygen vacancy density, the area with increasing electrons (red color) becomes larger around the titanium atoms, which indicates the increase in excess electrons derived from the oxygen vacancy. Moreover, when lithium ions are introduced into these anatase lattices with different oxygen vacancy densities, the differential charge densities are also calculated, as depicted in Fig. 8(e-1). The area with increasing electrons (red color) becomes larger around the lithium ions with the increase in the oxygen vacancy density, which implies the interaction of the lithium ions with the excess electrons. In other words, such excess electrons can act as centers of negative charge for the easy trapping of positive alkaline ions and even accelerate their diffusion process [45]. Furthermore, this implies that a high density of oxygen defects can ease the insertion and 
diffusion kinetic process of $\mathrm{Li}^{+}$into anatase and thus lead to more active sites, which is in accordance with the LIB electrochemical results.

Similarly, the differential charge density of sodium ion absorption on the anatase A (101) surface with different oxygen vacancy densities are calculated, as depicted in Fig. 9. Fig. 9(ad) shows the differential charge densities of the anatase (101) surface with different densities of oxygen vacancies. With the increasing oxygen vacancy density, the area with increasing electrons (red color) becomes larger around the adjacent titanium atoms with oxygen vacancy, which implies the increase in excess electrons derived from the oxygen vacancy. After the absorption of sodium ions on the surface of A (101) surface, as depicted in Fig. 9(e-f), the differential charge density undergoes redistribution, as depicted in Fig. $9(\mathrm{~g}-\mathrm{h})$. The electrons are absorbed on the surface of the sodium ions, indicating that excess electrons can be treated as centers of negative charges for the facile trapping of sodium ions. These results confirm the increase in the surface oxygen density, which accelerates the adsorption kinetic process of $\mathrm{Na}^{+}$ into the anatase surface, thus leading to more active sites, which is in accordance with the supercapacitor electrochemical results.

\section{Conclusions}

We have elucidated the structure-property relationship of the oxygen defect location and density with the surface and bulk charge storage behavior in TNTAs. Additionally, the surfaceexposed and bulk embedded oxygen defect evolution rule dependent on the annealing temperature is clarified for the first time. The remarkable enhancement of the bulk and surface charge storage behavior is caused by the increased bulk and surface oxygen defect densities, respectively. The $\mathrm{N}_{2}-600^{\circ} \mathrm{C}$-annealed TNTAs exhibit the highest capacity of $289.2 \mathrm{mAh} \mathrm{g}^{-1}$ at $0.8 \mathrm{C}$, and the $\mathrm{N}_{2}-900{ }^{\circ} \mathrm{C}$-annealed TNTAs yield an excellent specific capacitance of $35.6 \mathrm{mF}$ $\mathrm{cm}^{-2}$ and robust stability with over $96 \%$ capacitance retention after 10,000 cycles at a scan rate of $100 \mathrm{mV} \mathrm{s}^{-1}$. DFT calculations indicate that a high density of bulk oxygen defects can increase 
the excess electron density around the adjacent titanium atoms, which can interact with the lithium ions and enhance the electrons transportation to boost the bulk charge storage behavior. Meanwhile, surface oxygen defects in the anatase surface (101) plane also introduce excess electrons and enhance the surface potential, as also confirmed by the KPFM characterization, and the high density of surface oxygen defects accelerate the $\mathrm{Na}^{+}$adsorption kinetic process. Therefore, the surface charge storage behavior can be enhanced accordingly. The findings of this work provide fundamental insights into the role of surface/bulk defects in the activation of energy storage and serve as a novel strategy for significantly improving the energy storage efficiency through controlling the surface/bulk defect location and density of the electrode material. We believe that this strategy may also be applicable to other transitional metal oxides, such as $\mathrm{Fe}_{2} \mathrm{O}_{3}$ and $\mathrm{SnO}_{2}$. According to this tunable defect design strategy, one can thus rationally design a charge storage system to meet various demands in alkali metal ion batteries or supercapacitors and achieve better design through control of the defect location and density.

\section{Declaration of Competing Interest}

The authors declare that they have no competing financial interests.

\section{Acknowledgments}

This work was supported by the National Nature Science Foundation of China (11575025, U1832176), the Science and Technology Project of Beijing (Z171100002017008) and the Fundamental Research Funds for the Central Universities.

\section{Supporting Information}

Supporting Information is available from the Elsevier Online Library or from the author.

\section{References}


[1] Y. Liu, X.D. Yan, B.Q. Xu, J.L. Lan, Y.C. Liu, X.P. Yang, Y.H. Lin, C.W. Nan, J. Mater. Chem. A 47 (2018) 24298-24310.

[2] R. Schmuch, R. Wagner, G. Hörpel, T. Placke, M. Winter, Nat. Energy 4 (2018) 267-278.

[3] Q.Z. Zhang, D. Zhang, Z.C. Miao, X.L. Zhang, S.L. Chou, Small 14 (2018) 170288.

[4] Y.K. Wang, F.C., Z.X. Liu, Z.J. Tang, Q. Yang, Y. Zhao, S.Y. Du, Q. Chen, C.Y. Zhi, Angew. Chem. Int. Ed. 44 (2019) 15707-15711.

[5] X.X. Zuo, J. Zhu, P. M. Buschbaumb, Y.J. Cheng, Nano Energy 1 (2017) 113-143.

[6] G. Zubi, R.D. López, M. Carvalho, G. Pasaoglu, Renew. Sustain. Energy Rev. 89 (2018) $292-308$.

[7] Y. Wang, S.Y. Su, L.J. Cai, B.C. Qiu, N. Wang, J. Xiong, C. Yang, X.M. Tao, Y. Chai, Adv. Energy Mater. 9 (2019) 1900037.

[8] Y. Ma, C.P. Hou, H.P. Zhang, Q.Y. Zhang, H. Liu, S.D. Wu, Z. Guo, Electrochim. Acta 315 (2019) 114-123.

[9] S. Brutti, V. Gentili, H. Menard, B. Scrosati, P.G. Bruce, Adv. Energy Mater. 2 (2012) 322327.

[10] J. Chen, W.X. Song, H.S. Hou, Y. Zhang, M.J. Jing, X.N. Jia, X.B. Ji, Adv. Funct. Mater. 25 (2015) 6793-6801.

[11] L.Z. Wu, K. Zhang, X.F. Zhu, S.K. Cao, D.M. Niu, X.J. Feng, Langmuir 35 (2019) 51255129.

[12] J. Zhou, M. Guo, L.L. Wang, Y.B. Ding, Z.Z. Zhang, Y.H. Tang, C.B. Liu, S.L. Luo, Chem. Eng. J. 366 (2019) 163-171.

[13] C. Kim, S. Kim, J. Lee, J.Y. Kim, J. Yoon, ACS Appl. Mater. Interfaces 7 (2015) 74867491.

[14] N. T. Nguyen, S. Ozkan, I. Hwang, X.M. Zhou, P. Schmuki, J. Mater. Chem. A 5 (2017) 1895-1901. 
[15] M.C. Sun, Q.R. Xie, B. Li, J.W. Xiao, Z.D. Huang, Electrochim. Acta 363 (2020) 137201.

[16] S. Zhu, J.J. Li, X.Y. Deng, C.N. He, E.Z. Liu, F. He, C.S. Shi, N.Q. Zhao, Adv. Funct. Mater. 27 (2017) 1605017.

[17] C. Kim, S. Kim, S.P. Hong, J. Lee, J. Yoon, Phys. Chem. Chem. Phys. 18 (2016) 1437014375.

[18] N.A. Kyeremateng, ChemElectroChem 1 (2014) 1442-1466.

[19] W. Wei, G. Oltean, C. Wai Tai, K. Edstrom, F. Bjorefors, L. Nyholm, J. Mater. Chem. A $1(2013) 8160-8169$.

[20] N. Plylahan, M. Letiche, M. Kenza, S. Barr, B. Ellis, S. Maria, T.N.T. Phan, E. Bloch, P. Knauth, T. Djenizian, J. Power Sources 273 (2015) 1182-1188.

[21] X.B. Chen, L. Liu, P.Y. Yu, S.S. Mao, Science 331 (2011) 746-750.

[22] J.F. Zhang, Y. Wang, J.J. Wu, X. Shu, C.P. Yu, J.W. Cui, Y.Q. Qin, Y. Zhang, P.M. Ajayan, Y.C. Wu, Chem. Eng. J. 313 (2017) 1071-1081.

[23] N.S. Peighambardoust, S.K. Asl, R.Mohammadpour, S. K. Asl, Electrochim. Acta 270 (2018) 245-255.

[24] A.I. Savva, K.A. Smith, M. Lawson, S.R. Croft, A.E. Weltner, C.D. Jones, H.Bull, P.J. Simmonds, L. Li, H. Xiong, Phy. Chem. Chem. Phy. 20 (2018) 22537-22546.

[25] S. Mohajernia, P. Andryskov, G. Zoppellaro, S. Hejazi, S. Kment, R. Zboril, J. Schmidt, P. Schmuki, J. Mater. Chem. A 8 (2020) 1432.

[26] J.W. Chen, G.X. Wu, T.Y. Wang, X.D. Li, M.C. Li, Y.H. Sang, H. Liu, ACS Appl. Mater. Interfaces 9 (2017) 4634-4642.

[27] L.L. Hou, Z.J. Guan, M. Zhang, C.Q. He, Q.Y. Li, J.J. Yang, Catal. Sci. Technol. 8 (2018) $2809-2817$

[28] J. Chen, Y.L. Wu, K.L. Zhu, F. Sun, C.G. Guo, X.L. Wu, G.A. Cheng, R.T. Zheng, Ectrochim. Acta 316 (2019) 133-142. 
[29] J.H. Yan, Y.Y. Zhang, Y. Zhao, J. Song, S.H. Xia, S.J. Liu, J.Y. Yu, B. Ding, Sci. Adv. 6 (2020) 8538 .

[30] V. Perebeinosa, S.W. Chan, F. Zhang, Solid State Commun. 123 (2002) 295-297.

[31] B. Choudhury, A. Choudhury, Mater. Chem. Phy. 131 (2012) 666-671.

[32] S. Tsunekawa, K. Ishikawa, Z.Q. Li, Y. Kawazoe, A. Kasuya, Phys. Rev. Lett. 85 (2000) 3440

[33] J. Chen, Y.L. Fu, F. Sun, Z.G. Hu, X. Wang, T. Zhang, F.S. Zhang, X.L. Wu, H.S. Chen, G.A. Cheng, R.T. Zheng, Chem. Eng. J. 400 (2020) 125784.

[34] R. Schmitt, A. Nenning, O. Kraynis, R. Korobko, A.I. Frenkel, I. Lubomirsky, S.M. Hailef, J. M. Rupp, Chem. Soc. Rev. 49 (2020) 554.

[35] J.W. Chen, B. Luo, Q.S. Chen, F. Li, Y.J. Guo, T. Wu, P. Peng, X. Qin, G.X. Wu, M.Q. Cui, L.H. Liu, L.H. Chu, B. Jiang, Y.F. Li, X.Q. Gong, Y. Chai, Y.P. Yang, Y.H. Chen, W. Huang, X.G. Liu, M.C. Li, Adv. Mater. 32 (2020) 1905578.

[36] L. Nie, Y.F. Li, S.J. Chen, K. Li, Y.Q. Huang, Y.B. Zhu, Z.T. Sun, J.C. Zhang, Y.J. He, M.K. Cui, S.C. Wei, F. Qiu, C. Zhong, W. Liu, ACS Appl. Mater. Interfaces 11 (2019) $32373-32380$.

[37] X.S. Wu, S.X. Xia, Y.Q. Huang, X.C. Hu, B. Yuan, S.J. Chen, Y. Yu, W. Liu, Adv. Funct. Mater. 29 (2019) 1903961.

[38] N.N. Li, L. Sun, K. Wang, S. Xu, J. Zhang, X.X. Guo, X.H. Liu, J. Energy Chem. 51 (2020) $62-71$.

[39] Z. Shen, L. Cao, C. D. Rahn, C.Y. Wang, J. Electrochem. Soc. 160 (2013) 1842-1846.

[40] Q. Ni, Y. Bai, Y. Li, L.M. Ling, L.M. Li, G.H. Chen, Z.H. Wang, H.X. Ren, F. Wu, C. Wu, Small 14 (2018) 1702864.

[41] Y. Li, X.Z. Zhou, Y. Bai, G.H. Chen, Z.H. Wang, H. Li, F. Wu and C. Wu. ACS Appl. Mater. Interfaces 9 (2017) 19852-19860. 
[42] N. Zhu, F. Wu, Z.H. Wang, L.M. Ling, H. Yang, Y.N. Gao, S.N. Guo, L.M. Suo, H. Li, H.J. Xue, Y. Bai, C. Wu, J. Energy Chem. 51 (2020) 72-80.

[43] Z.V. Bobyleva, O.A. Drozhzhin, K.A. Dosaev, A. Kamiyama, S.V. Ryazantsev, S. Komaba, E.V. Antipov, Electrochim. Acta 354 (2020) 136647.

[44] Y. Li, J. Qian, M.H. Zhang, S. Wang, Z.H. Wang, M.S. Li, Y. Bai, Q.Y. An, H.J. Xu, F. Wu, L.Q. Mai, C. Wu, Adv. Mater. 32 (2020) 2005802.

[45] Y.Q. Zhang, L. Tao, C. Xie, D.D. Wang, Y.Q. Zou, R. Chen, Y.Y. Wang, C.K. Jia, S.Y. Wang, Adv. Mater. 32 (2020) 1905923.

[46] H.K. Wang, T.H. Yao, C. Li, L.J. Meng, Y.H. Cheng, Chem. Eng. J. 397 (2020) 125385.

[47] Z. Li, Y.T. Ding, W.J. Kang, C. Li, D. Lin, X.Y. Wang, Z.W. Chen, M.H. Wu, D.Y. Pan, Electrochim. Acta 161 (2015) 40-47.

[48] H. Zhou, Y.R. Zhang, J. Phys. Chem. C 118 (2014) 5626-5636.

[49] M. Salari, S.H. Aboutalebi, A.T. Chidembo, I.P. Nevirkovets, K. Konstantinov, H.K. Liu, Phys. Chem. Chem. Phys. 14 (2012) 4770-4779.

[50] Z.R. Zhang, Z.P. Yao, Y.Q. Meng, D.Q. Li, Q.X. Xia, Z.H. Jiang, Inorg. Chem. 58 (2019) $1591-1598$.

[51] X.H. Lu, G.M. Wang, T. Zhai, M.H. Yu, J.Y. Gan, Y.X. Tong, Y. Li, Nano Lett. 12 (2012) 1690-1696.

[52] H. Masuda, K. Matsushita, D. Ito, D. Fujita, N. Ishida, Commun. Chem. 2 (2019) 1-6.

[53] W.N. Zhan, M. Zhu, J.L. Lan, H.C. Yuan, H.J. Wang, X.P. Yang, G. Sui, ACS Appl. Mater. Interfaces 12 (2020) 51488-51498. 


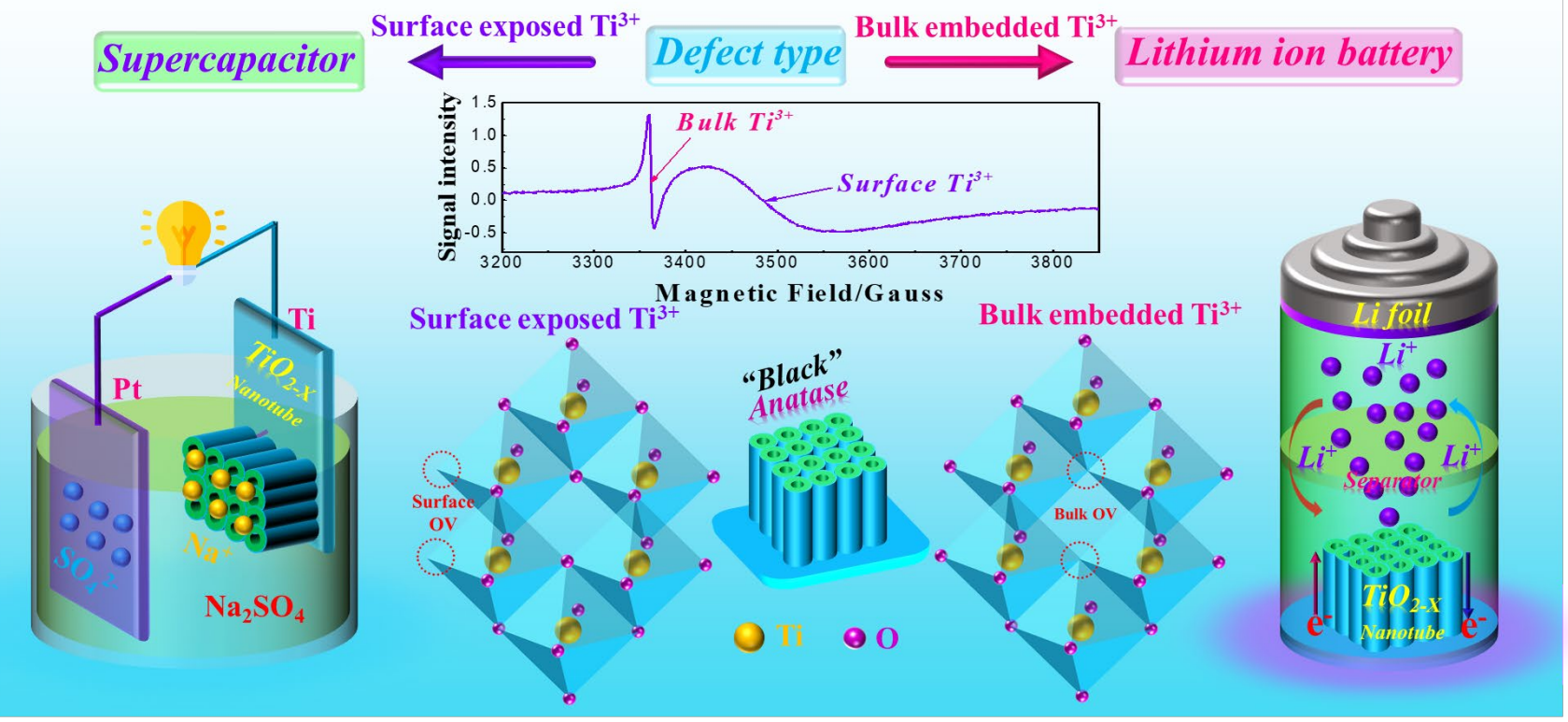

Scheme 1. Defect design strategy for performance enhancement of lithium-ion battery and supercapacitor 

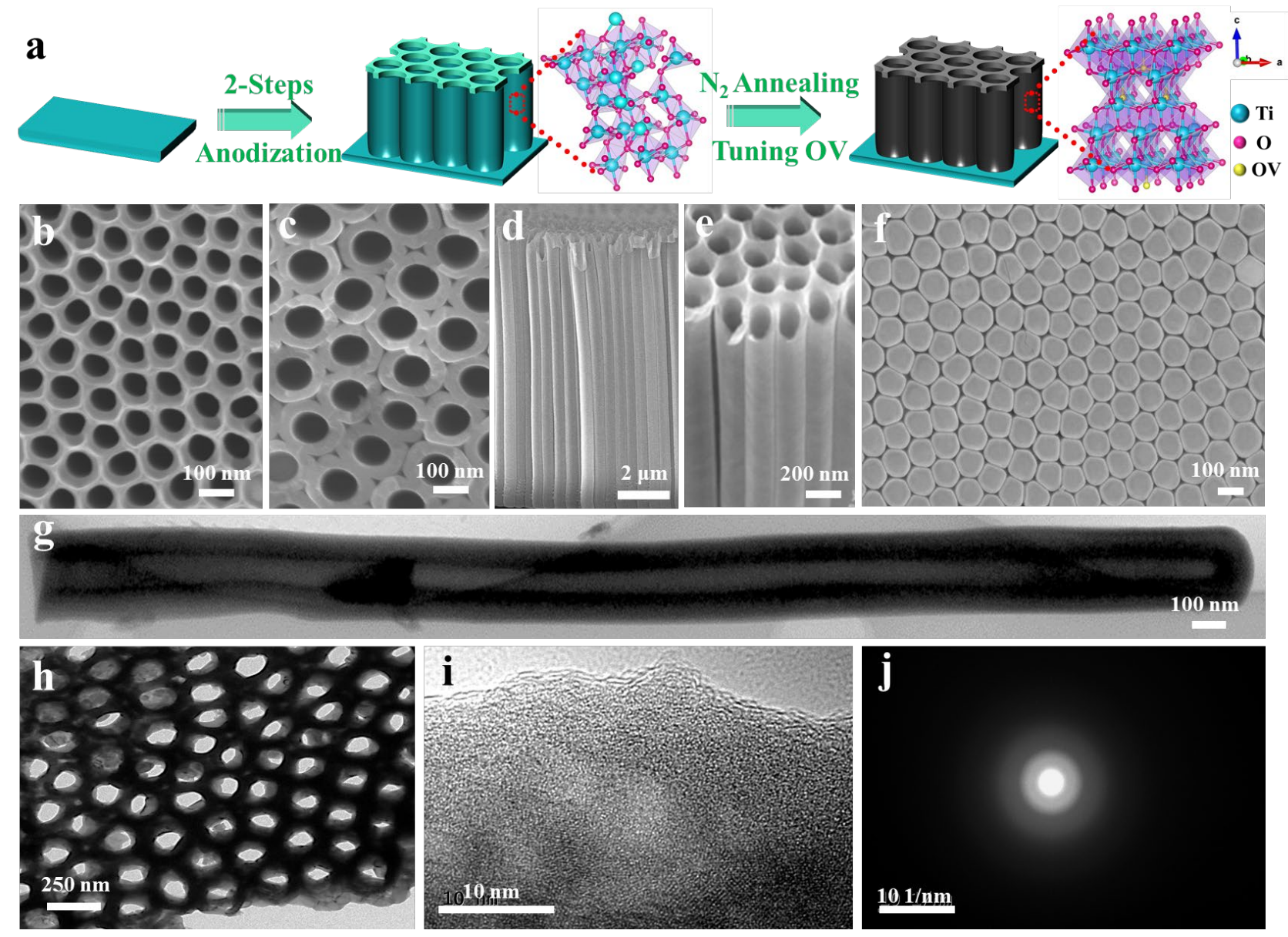

Fig. 1. (a) Synthesis procedure of black TNTAs; SEM images of the as anodized TNTAs (b)

surface, (c) sub-surface, (d) cross-section, (e) enlarged cross-section and (f) bottom. TEM images of the as anodized TNTAs of (g) cross-section; (h) surface; (i) HRTEM image of the surface; (j) SAED image corresponding to (g). 

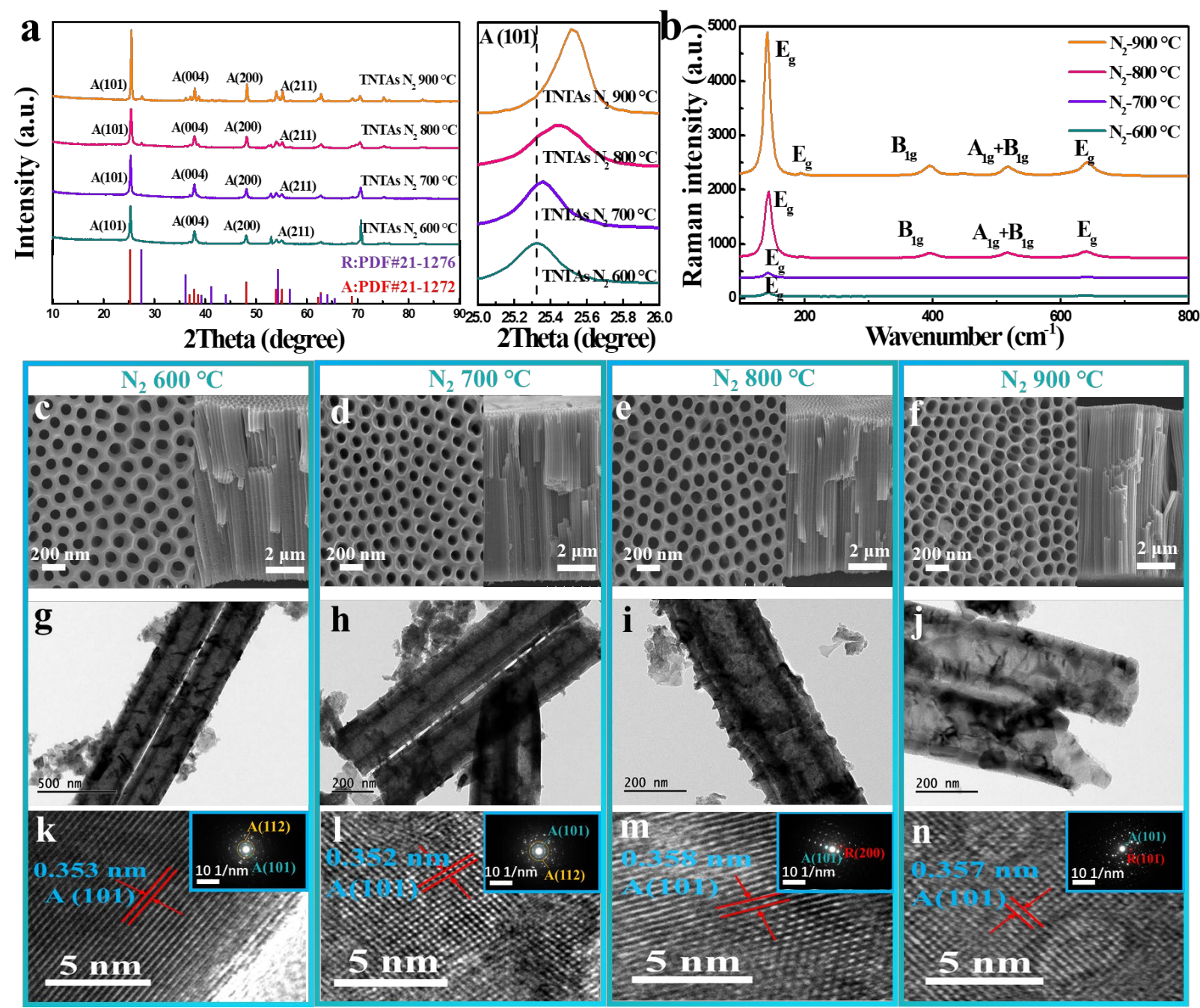

Fig. 2. (a) XRD patterns and the enlarged (101) peaks and (b) corresponding Raman spectra of TNTAs annealed at $600{ }^{\circ} \mathrm{C}, 700{ }^{\circ} \mathrm{C}, 800{ }^{\circ} \mathrm{C}$, and $900{ }^{\circ} \mathrm{C}$ in $\mathrm{N}_{2}$; SEM images of the samples annealed in $\mathrm{N}_{2}$ at (c) $600{ }^{\circ} \mathrm{C}$; (d) $700{ }^{\circ} \mathrm{C}$; (e) $800{ }^{\circ} \mathrm{C}$; (f) $900{ }^{\circ} \mathrm{C}$; the insets are the cross section SEM images of corresponding TNTAs. TEM images of the samples annealed in $\mathrm{N}_{2}(\mathrm{~g}) 600{ }^{\circ} \mathrm{C}$; (h) $700{ }^{\circ} \mathrm{C}$; (i) $800{ }^{\circ} \mathrm{C}$; (j) $900{ }^{\circ} \mathrm{C}$; HRTEM images of the samples annealed in $\mathrm{N}_{2}$ (k) $600{ }^{\circ} \mathrm{C}$; (l) $700{ }^{\circ} \mathrm{C}$; (m) $800{ }^{\circ} \mathrm{C}$; (n) $900{ }^{\circ} \mathrm{C}$; the insets are the corresponding SAED images. 

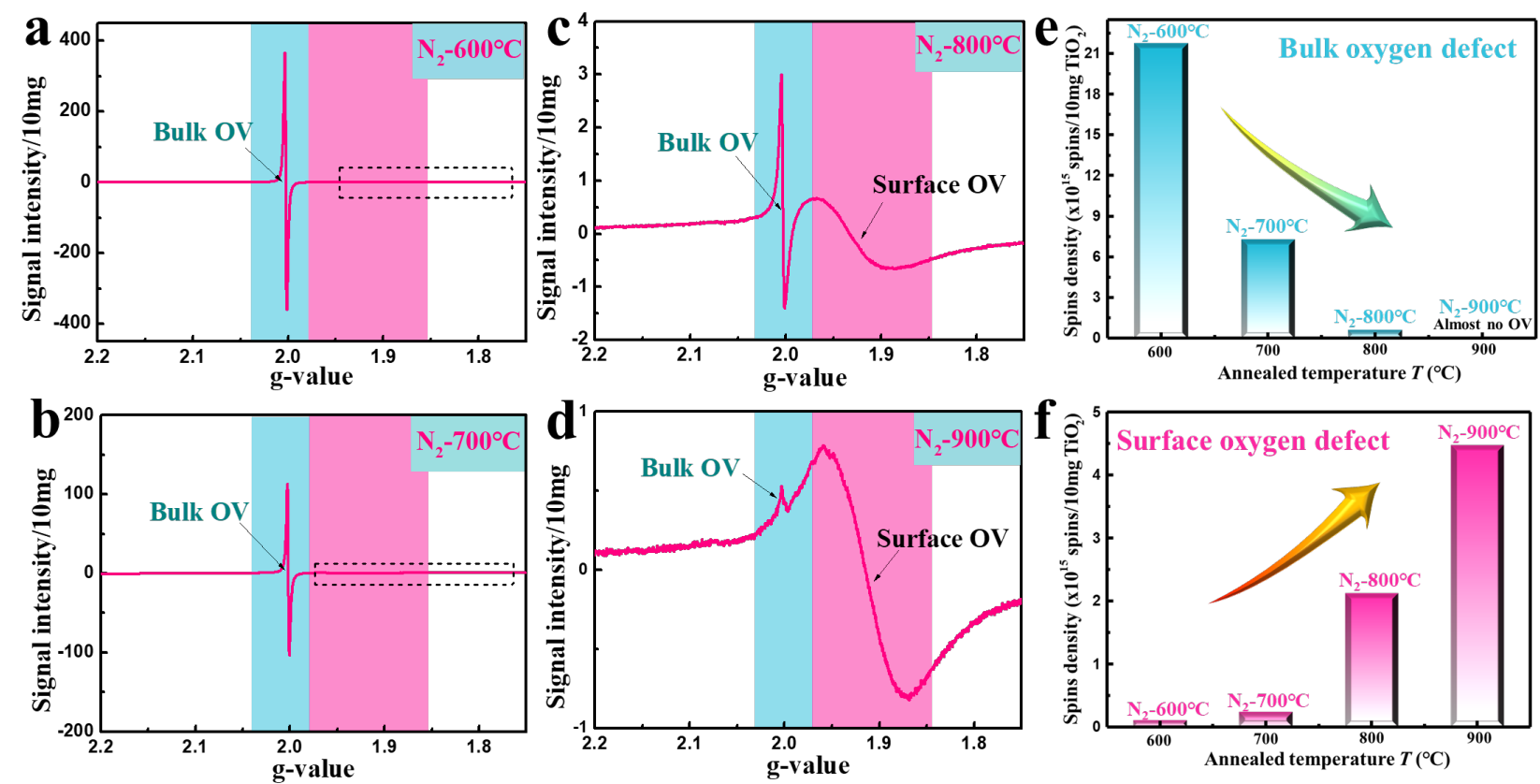

Fig. 3. EPR spectra of TNTAs annealed in $\mathrm{N}_{2}$ (a) $600{ }^{\circ} \mathrm{C}$; (b) $700{ }^{\circ} \mathrm{C}$; (c) $800{ }^{\circ} \mathrm{C}$; (d) $900{ }^{\circ} \mathrm{C}$; spin density of all the samples (e) bulk oxygen vacancy; (f) surface oxygen vacancy. 

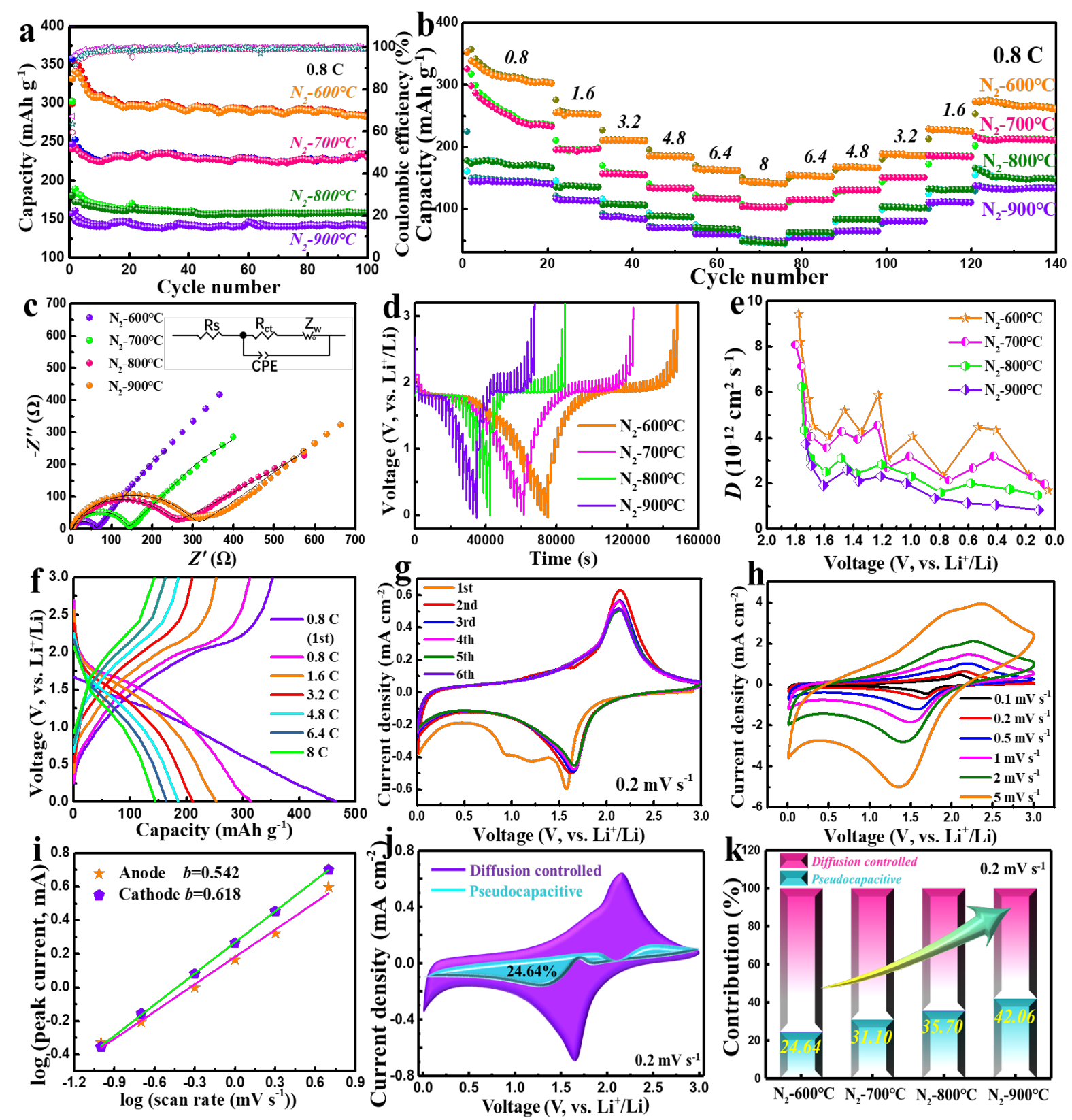

Fig. 4. Lithium-ion storage performance of TNTAs annealed in $\mathrm{N}_{2}$ at $600{ }^{\circ} \mathrm{C}, 700{ }^{\circ} \mathrm{C}, 800{ }^{\circ} \mathrm{C}$, and $900{ }^{\circ} \mathrm{C}$. (a) Cyclic performance at $0.8 \mathrm{C}$; (b) rate performance; (c) EIS plots; (d) GITT curves; (e) corresponding lithium-ion diffusion coefficients; (f) GCD plots of the rate cycling of TNTAs annealed in $\mathrm{N}_{2}$ at $600{ }^{\circ} \mathrm{C}$. (g) Initial 6 cycles of $\mathrm{N}_{2}-600{ }^{\circ} \mathrm{C}$ TNTA-based lithium-ion battery. Pseudocapacitive analysis of $\mathrm{N}_{2}-600{ }^{\circ} \mathrm{C}$ TNTAs; (h) CV curves with different scan rates; (i) calculated $b$ values; (j) pseudocapacitive contribution plot at $0.2 \mathrm{mV} \mathrm{s}^{-1}$; (k) pseudocapacitive contributions at $0.2 \mathrm{mV} \mathrm{s}^{-1}$ of $\mathrm{N}_{2}-600{ }^{\circ} \mathrm{C}, 700{ }^{\circ} \mathrm{C}, 800{ }^{\circ} \mathrm{C}$, and $900{ }^{\circ} \mathrm{C}-$ annealed samples. 

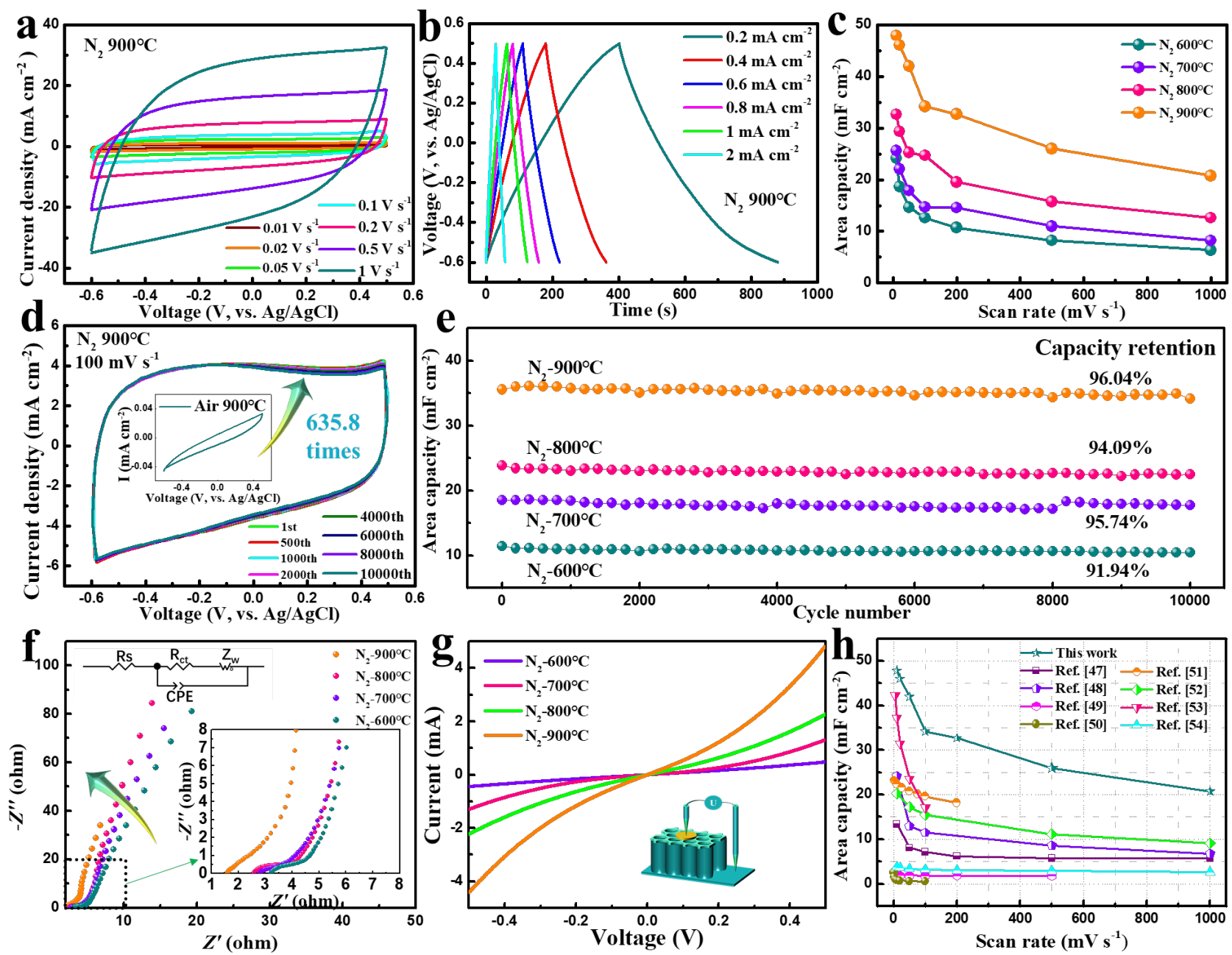

Fig. 5. Supercapacitor behavior of the $\mathrm{N}_{2}-900{ }^{\circ} \mathrm{C}$ sample (a) $\mathrm{CV}$ curves at different scan rates; (b) GCD plots under various current densities; (c) rate performance of the $\mathrm{N}_{2}$ $600{ }^{\circ} \mathrm{C} / 700{ }^{\circ} \mathrm{C} / 800{ }^{\circ} \mathrm{C} / 900{ }^{\circ} \mathrm{C}$ samples; (d) cycling life at scan rate of $100 \mathrm{mV} \mathrm{s}^{-1}$; (e) cycling capacity retention ability at scan rate of $100 \mathrm{mV} \mathrm{s}^{-1}$; (f) EIS plots of $\mathrm{N}_{2-}$ $600{ }^{\circ} \mathrm{C} / 700{ }^{\circ} \mathrm{C} / 800{ }^{\circ} \mathrm{C} / 900{ }^{\circ} \mathrm{C}$ sample-based supercapacitor. Electronic conductivity measured from $(\mathrm{g})$ solid-state electronic conductivity $I-V$ plots; (h) summary of the $\mathrm{TiO}_{2}$ nanotube electrode-based supercapactor performance in the literature. 

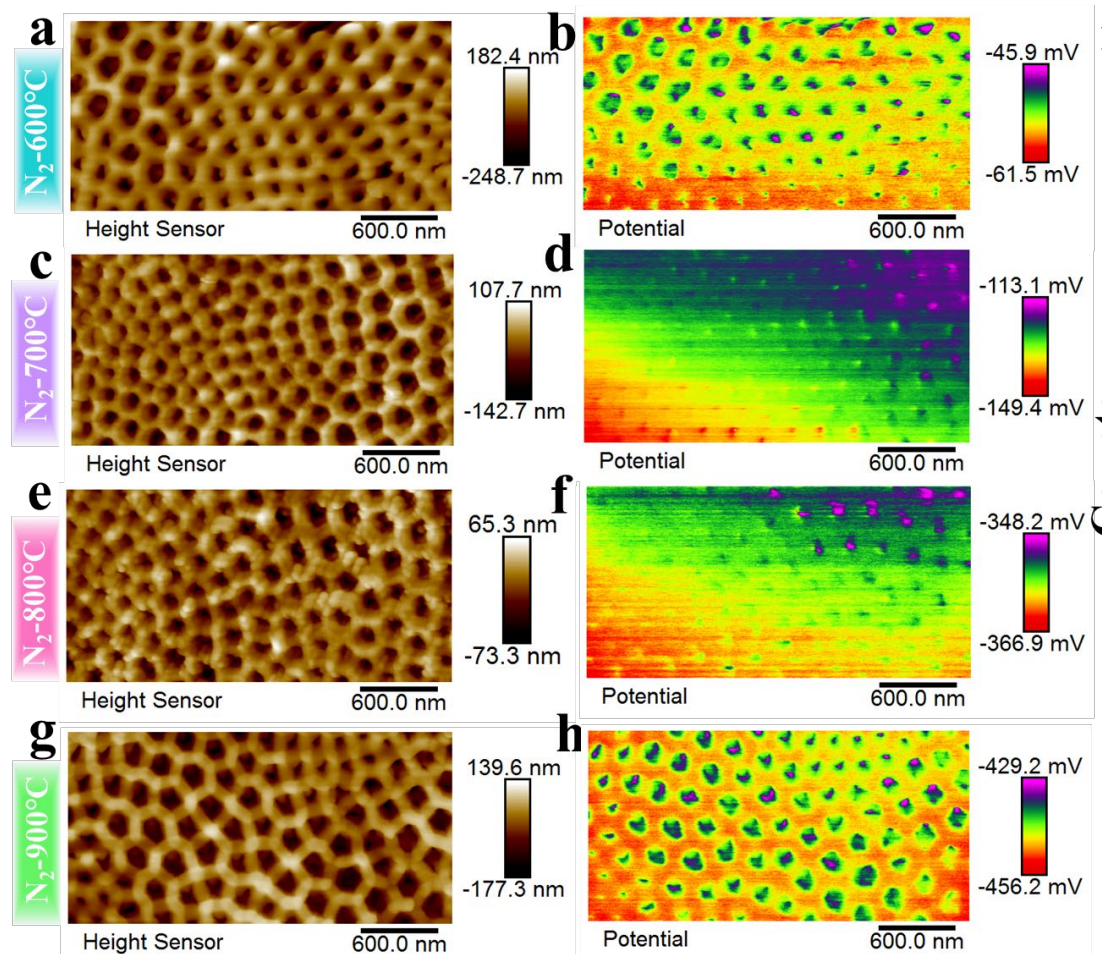

h

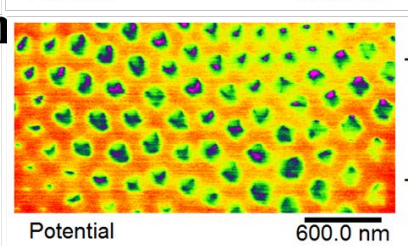

$\overline{600.0 \mathrm{~nm}}$

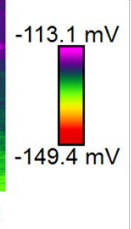

Potential

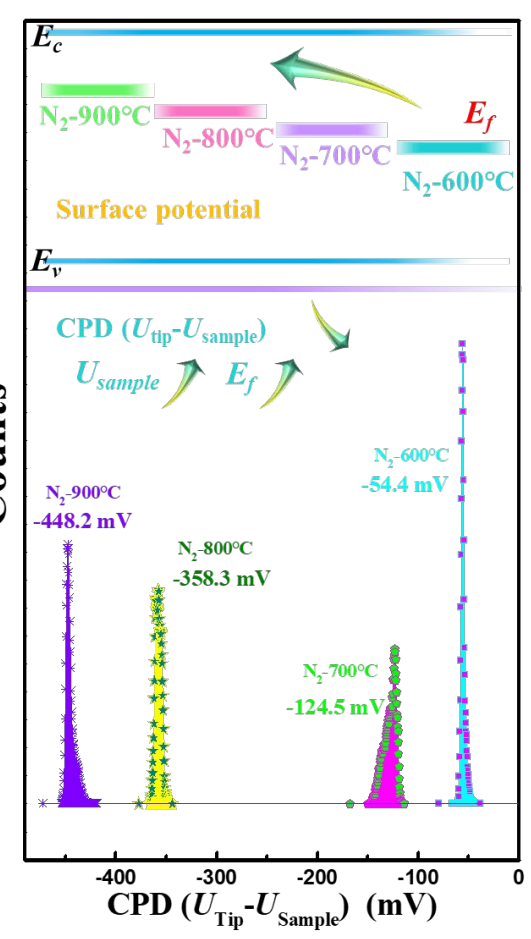

Fig. 6. KPFM characterization of surface potential and corresponding surface morphology of (a and b) $\mathrm{N}_{2}-600{ }^{\circ} \mathrm{C}$; (c and d) $\mathrm{N}_{2}-700{ }^{\circ} \mathrm{C}$; (e and f) $\mathrm{N}_{2}-800{ }^{\circ} \mathrm{C}$; (g and h) $\mathrm{N}_{2}-900{ }^{\circ} \mathrm{C}$. (i) Overall potential of the four types of TNTAs. 

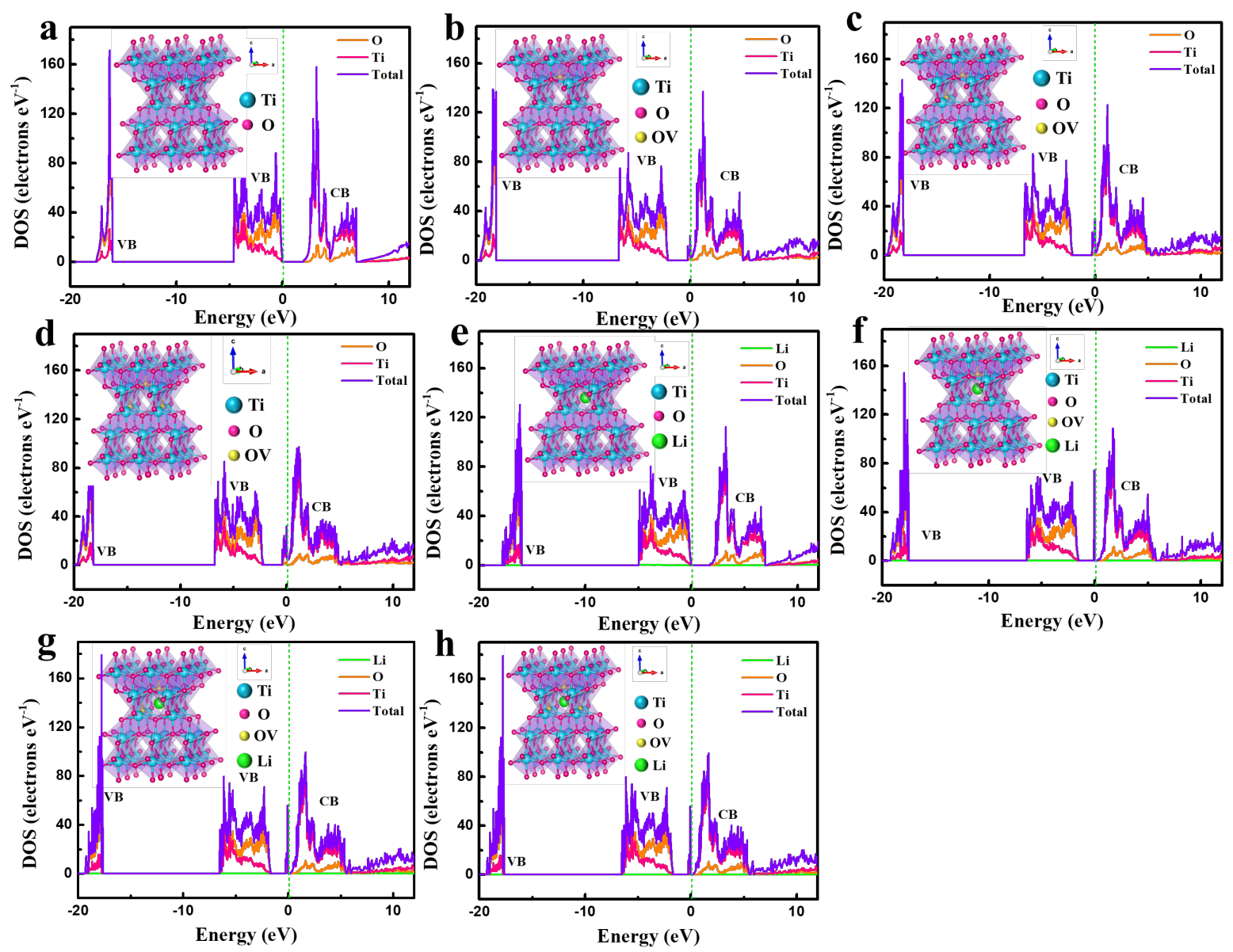

Fig. 7. (a-d) Calculated density of states (DOSs) anatase $\mathrm{TiO}_{2}$ with different oxygen defect densities. (e-h) Calculated DOSs of lithiation anatase $\mathrm{TiO}_{2}$ with different oxygen defect densities. The insets display corresponding crystals structures. 

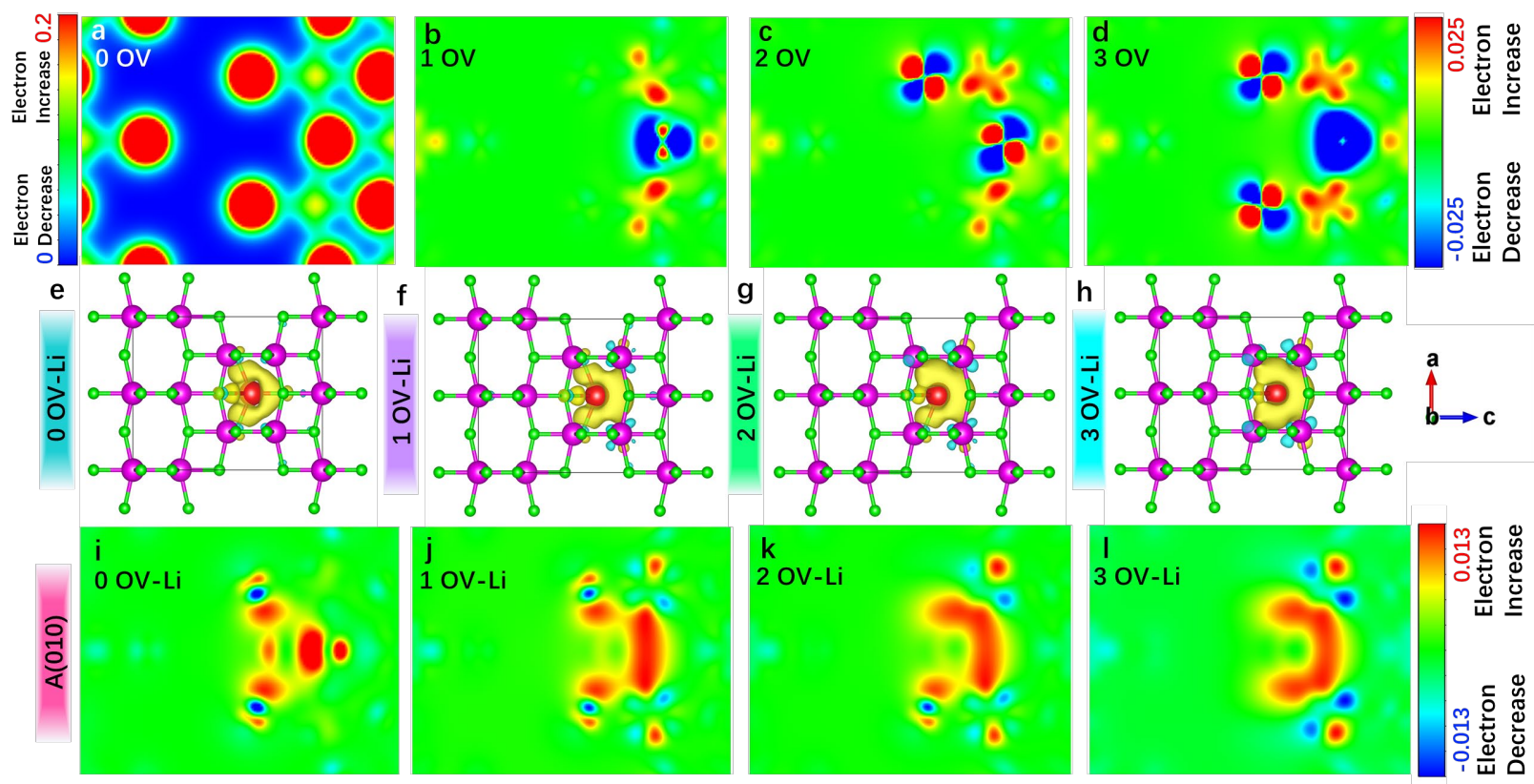

Fig. 8. (a) Charge density of stoichiometric ratio anatase $\mathrm{TiO}_{2}$; (b-d) differential charge density of anatase $\mathrm{TiO}_{2}$ with different concentrations of oxygen vacancies; (e-h) differential charge density of lithiation anatase $\mathrm{TiO}_{2}$ with different concentrations of oxygen vacancies; (i-1) A (010) plane differential charge density of lithiation anatase $\mathrm{TiO}_{2}$ with different concentrations of oxygen vacancies (Titanium atom: pink; oxygen atom: green; $\mathrm{Li}^{+}:$red). 
a
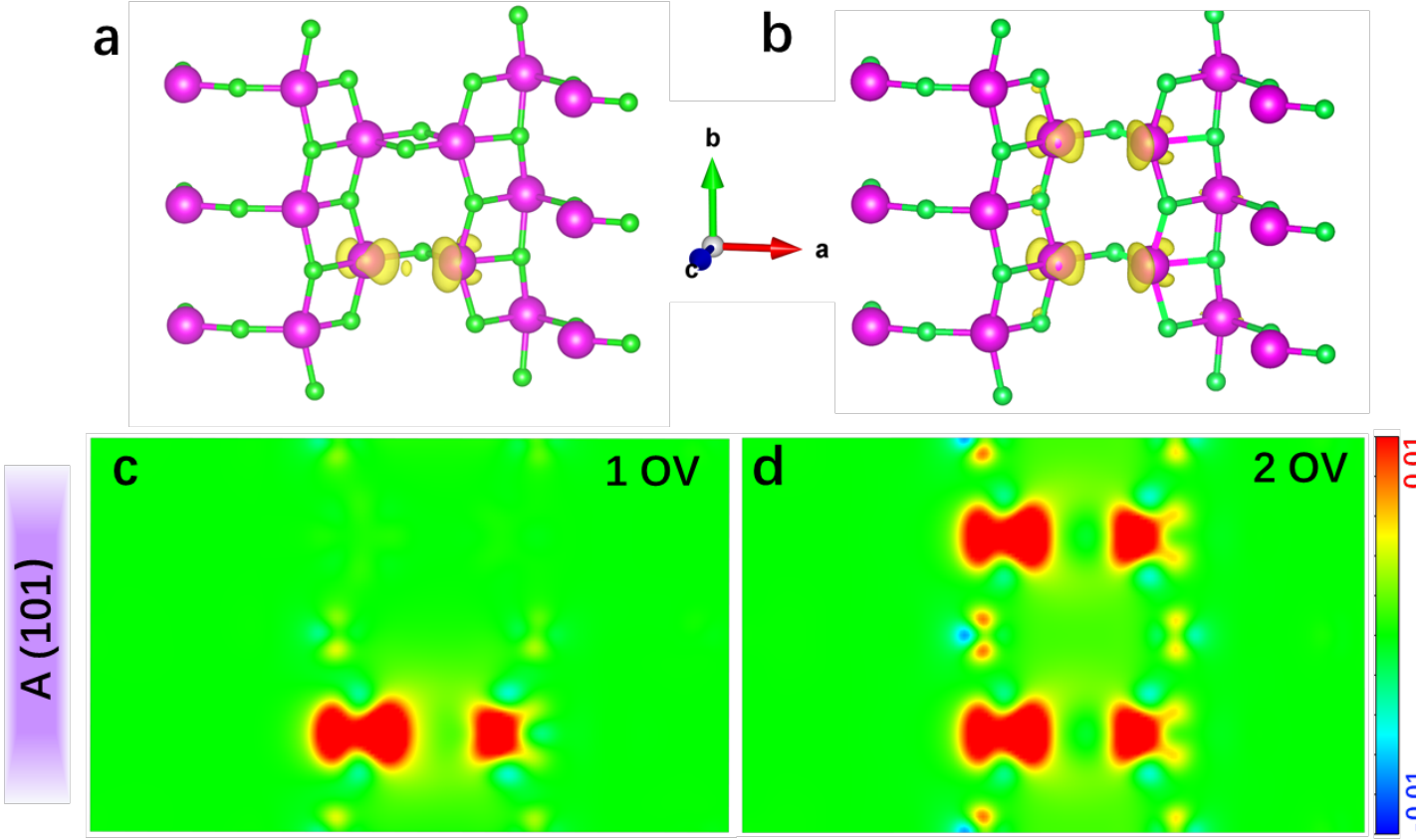

造

e

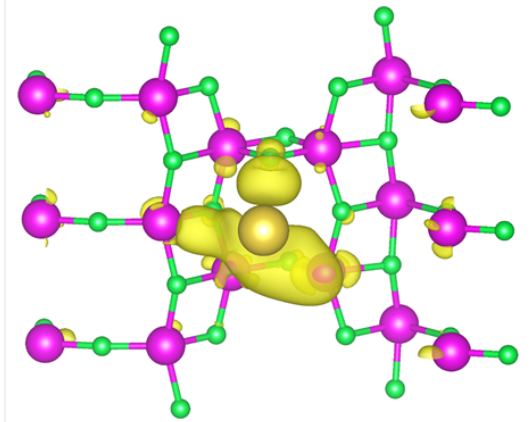

f
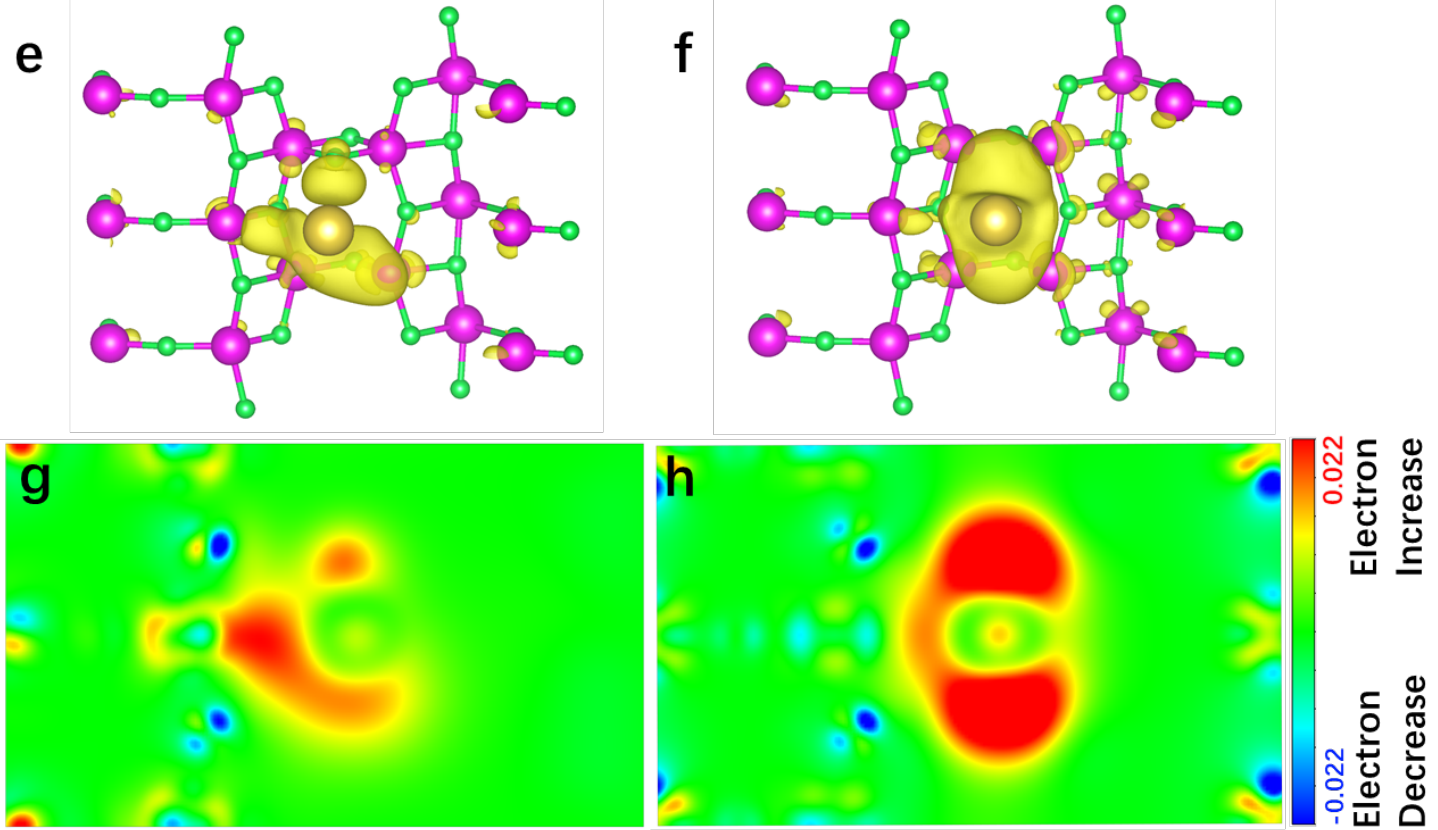

Fig. 9. Differential charge density of anatase (101) surface with (a) one oxygen vacancy and (b) two oxygen vacancies; A (101) plane differential charge density with (c) one oxygen vacancy; (d) with two oxygen vacancies; differential charge density of anatase (101) surface with (e) one oxygen vacancy absorbed by one $\mathrm{Na}^{+}$; (f) two oxygen vacancies absorbed by one $\mathrm{Na}^{+}$; A (101) plane differential charge density with (g) one oxygen vacancy absorbed by one $\mathrm{Na}^{+}$; (h) two oxygen vacancies absorbed by one $\mathrm{Na}^{+}$(Titanium atom: pink; oxygen atom: green; $\mathrm{Na}^{+}$:yellow). 


\section{Graphic abstract}

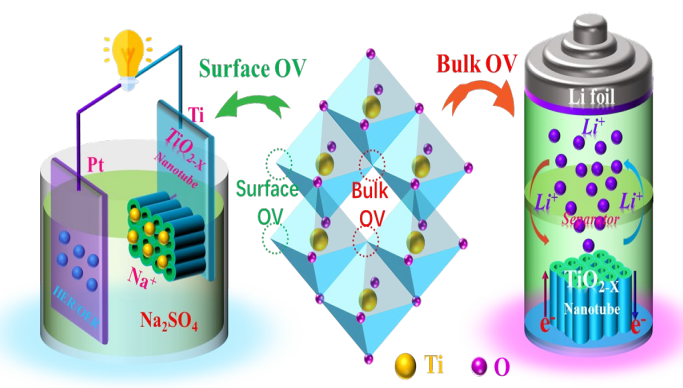

Oxygen defect location and density can be tuned in $\mathrm{TiO}_{2}$ nanotube arrays and the excess electrons originated from the oxygen defect can enhance performance of lithium ion battery and supercapacitor simultaneously. 